\title{
Countermeasure for Round Trip Delay Which Occurs in Between Satellite and Ground with Software Network Accelerator
}

\author{
Kohei Arai \\ Graduate School of Science and Engineering \\ Saga University \\ Saga City, Japan
}

\begin{abstract}
Countermeasure for round trip delay which occurs in between satellite and ground with network accelerator is investigated together with operating system dependency on effectiveness of accelerator. Also disaster relief data transmission experiments are conducted for mitigation of disaster together with acceleration of disaster related data transmission between local government and disaster prevention center. Disaster relief information including remote sensing satellite images and information from the disaster occurred areas to local government for creation of evacuation information is accelerated so that it becomes possible to send them to the residents in the suffered areas due to disaster through data broadcasting in the digital TV channel.
\end{abstract}

Keywords-IP comunication through Internet Satellite; WINDS; software accerelator.

\section{INTRODUCTION}

Wideband Inter-Networking engineering test and Demonstration Satellite: WINDS (KIZUNA ${ }^{1}$ in Japanese) was launched on February 23, 2008 (JST) from the Tanegashima Space Center (one of the launch sites in Japan) to establish the world's most advanced information and telecommunications network. It is expected that this information and telecommunications network's speed and capacity will be much higher than anything achieved previously.

KIZUNA satellite communication system aims for a maximum speed of $155 \mathrm{Mbps}$ (receiving) / 6Mbps (transmitting) for households with 45-centimetre aperture antennas (the same size as existing Communications Satellite antennas), and ultra-high speed $1.2 \mathrm{Gbps}$ communication for offices with five-meter antennas. In addition to establishing a domestic ultra high speed Internet network, the project also aims to construct ultra high speed international Internet access, especially with Asian Pacific countries and regions that are more closely related to Japan.

KIZUNA project is responsible for the demonstration of the validity and usefulness of technologies related to large-capacity data communications in our space infrastructure project, "iSpace," ${ }^{2}$ the purpose of which is to promote the use of satellites

\footnotetext{
${ }^{1}$ http://www.jaxa.jp/countdown/f14/overview/kizuna_e.html

${ }^{2}$ http://ja.wikipedia.org/wiki/I-Space
}

in such fields as Internet communications, education, medicine, disaster measures and Intelligent Transport Systems.

One of the problems on KIZUNA is around $0.8 \mathrm{sec}$ of the delay time of round trip delay which occurs in between satellite and ground ( $0.3 \mathrm{sec}$ by the factor of two) and the delay time in Asynchronous Transfer Mode: ATM switch ${ }^{3}$ onboard KIZUNA satellite $(0.2 \mathrm{sec})$. Because KIZUNA provides IP communications based on TCP/IP protocol ${ }^{4}$ so that shake-hand protocol including acknowledge confirmation is required results in effective network speed (data rate) is degraded due to the delay time.

In order to overcome such problem, hardware type of accelerator such as Sky- $\mathrm{X}^{5}$, Blue coat ${ }^{6}$ is developed which is a little bit expensive though. In this paper, software type of accelerator $^{7}$ is proposed with some evidences of experimental results which are obtained with KIZUNA satellite. Through experiments with KIZUNA satellite, performance of the proposed software TCP accelerator is confirmed [1]-[9]. Such accelerator is useful for not only satellite communication but also the other surface communications with TCP/IP protocol communications.

The following section describes the proposed software accelerator followed by some experimental results. Then concluding remarks with some discussions is followed by.

\section{PROPOSED SOFTWARE ACCERELATOR}

\section{A. Problem Due to Delay}

Under the TCP/IP protocol, sender transmits a shake-hand message first with header information including packet size, sender address, etc., and then receiver replies with $\mathrm{ACK}^{8}$ : Acknowledgement. After that sender send packets as shown in Figure 1.

\footnotetext{
${ }^{3}$ http://en.wikipedia.org/wiki/Asynchronous_Transfer_Mode

${ }^{4} \mathrm{http} / / / \mathrm{www}$. comptechdoc.org/independent/networking/protocol/protnet.html

${ }^{5} \mathrm{http}: / /$ www.1st-computer-networks.co.uk/packeteerSkyXAccelerator250.php

${ }^{6} \mathrm{http}: / / \mathrm{www} \cdot$ bluecoat.com/

${ }^{7} \mathrm{http} / / / \mathrm{www}$.viasat.com/government-communications/information-

assurance/compact-xpep

http://www.bitpipe.com/tlist/TCP/IP-Acceleration.html

${ }^{8} \mathrm{http}: / /$ en.wikipedia.org/wiki/Transmission_Control_Protocol
} 


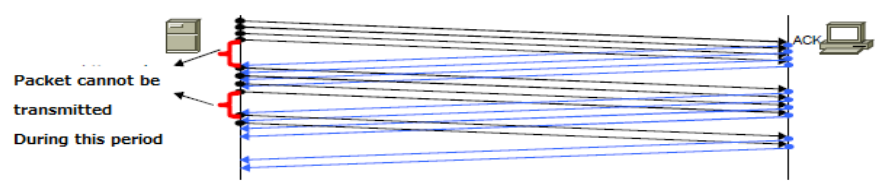

Figure 1. Communication procedure of TCP/IP protocol

If there is some delay in the network links, then packet cannot be sent during the red portions of period in Figure 1. Thus effective data rate is somewhat degraded depending on window size and delay time. The typical round trip time (Delay time) is $5 \mathrm{~ms}$ for Local Area Network: LAN, $10 \mathrm{~ms}$ for Metropolitan Area Network: MAN, 100ms for Wide Area Network: WAN and $600 \mathrm{~ms}$ for geostationary satellites as well as $1200 \mathrm{~ms}$ for geostationary satellite double hop. Throughput for $5 \mathrm{~ms}$ delay of LAN with $8 \mathrm{~KB}$ of window size is $12.8 \mathrm{Mbps}$ while that with $32 \mathrm{~KB}$ window size is $51.2 \mathrm{Mbps}$. Meanwhile, throughput for $10 \mathrm{~ms}$ delay of MAN with $8 \mathrm{~KB}$ window size is $64 \mathrm{Mbps}$ while that with $32 \mathrm{~KB}$ window size is $25.6 \mathrm{Mbps}$. On the other hands, throughput for $100 \mathrm{~ms}$ delay of WAN with $8 \mathrm{~KB}$ window size is $640 \mathrm{Kbps}$ while that with $32 \mathrm{~KB}$ window size is $2.6 \mathrm{Mbps}$. Furthermore, throughput for $600 \mathrm{~ms}$ delay of geostationary satellite link with $8 \mathrm{~KB}$ window size is $107 \mathrm{Kbps}$ while that with $32 \mathrm{~KB}$ is $427 \mathrm{Kbps}$ and throughput for $1200 \mathrm{~ms}$ delay of geostationary double hop link with $8 \mathrm{~KB}$ window size is $53 \mathrm{Kbps}$ while that with $32 \mathrm{~KB}$ window size is $213 \mathrm{Kbps}$. In accordance with window size, throughput is increased. Meanwhile throughput is decreased with increasing of delay time.

\section{B. Hardware Accelerator}

TCP protocol has three major merits, error corrections, endto-end flow control and collision avoidance control so that the most of IP communication adopts TCP protocol. Remaining such these merits, TCP is converted to XTP (Xpress Transport Protocol) which is appropriate protocol for high speed of long distance communications. Sky- $\mathrm{X}^{9}$ adopts $\mathrm{XTP}^{10}$ and TCP accelerations. XTP includes (1) Dynamic window sizing, (2) Rate control and flow control, (3) Selective retransmission mechanism, (4) Data compressions, and (5) HTTP acceleration ${ }^{11}$. Meanwhile, TCP acceleration is based on SCPS $^{12}$ : Space Communication Protocol Standard of MILSTD-2045-4000 ${ }^{13}$.

\section{Software Accelerator}

Packets on the network flow have to be handled by software accelerator. LAN driver software has to be modified to accelerate together with some modifications of LAN board. Free open source software of virtual LAN driver is used for the proposed software accelerator. It is known that all the functions of TCP protocol have not been realized with the virtual LAN driver. Therefore, the proposed software accelerator may choose functionalities TCP using the control panel shown in

\footnotetext{
${ }^{9}$ http://www.netone.co.jp/seminar/tfa9q100000031eb-att/06DSE_SkyX.pdf

${ }^{10} \mathrm{http}: / /$ www.sophia-it.com/content/Xpress+Transport+Protocol

${ }^{11}$ http://en.wikipedia.org/wiki/Varnish_(software)

12 http://www.scps.org/

$13 \mathrm{http} / / /$ www.everyspec.com/MIL-STD/MIL-STD+(2000+-+2999)/MILSTD-2045_47001C_25099/

http://www.gwg.nga.mil/ntb/baseline/docs/44500/index.html
}

Figure 2. Thus efficiency or effective data rate, throughput, etc. can be measured with the selected functions, in particular for status of the buffer memory.

The most important function of the proposed software accelerator is an acknowledge handling. Delay time can be shortened because network performance is degraded due to delay time. Although it is not possible that physical distance is shortened, the required time for acknowledge sending can be shortened. Actually, acknowledge is replied immediately after receiving TCP packet on the virtual LAN driver ${ }^{14}$. The actual data packet can be notified to the receiver with the different protocol. Thus acknowledge can be replied immediately after receiving TCP packet so that delay time can be shortened remarkable (ideally, delay time is going to be zero). Although this method may avoid the delay time induced problems, the actual data packets have to be sent to the receiver certainly.

Maximum buffer size for receiving has to be noticed. TCP protocol of automatic tuning of receiving buffer size based on RFC1 $323^{15}$ and Windows Vista may control buffer size for increasing effective data rate (maximum buffer size for sending is also to be notified for Windows Vista ${ }^{16}$ of automatic tuning). The receiving buffer size does not include data packet which are not yet received by application layer. In case of IP communication through KIZUNA, delay time is $0.8 \mathrm{sec}$ so that all the data packets which are not yet received by application layer can be processed with the current performance of personal computers. That is the same thing for the other communication satellite in the geostationary orbit (Delay time is $0.6 \mathrm{sec})$. Therefore, available receiving buffer size cannot be sent properly. The proposed software accelerator rewrites the available receiving buffer size is replaced to the maximum receiving buffer size.

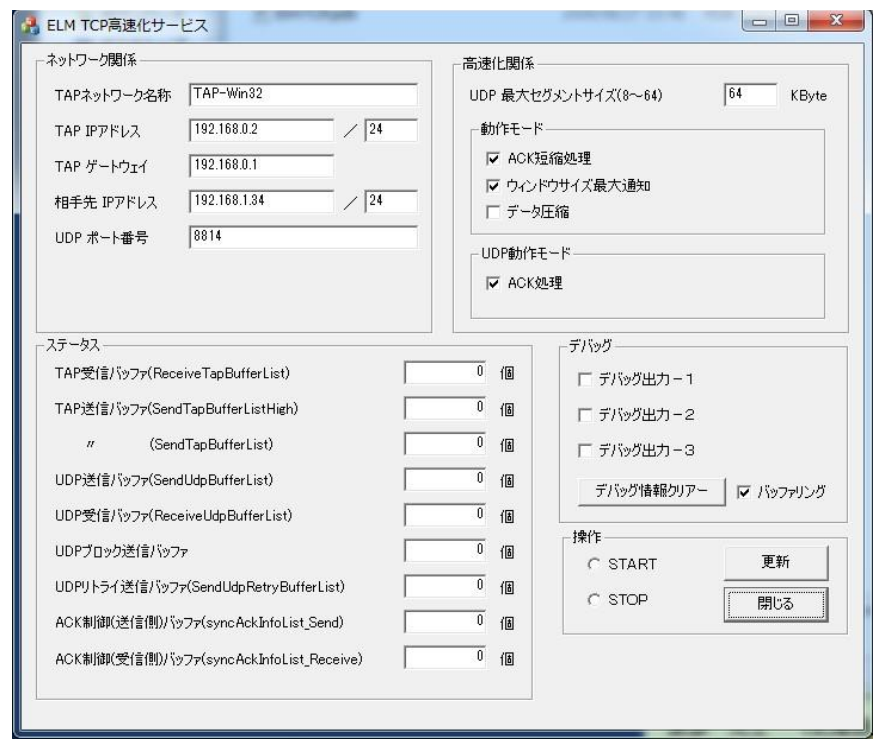

Figure 2. Control panel for selection of functionalities of TCP protocol.

\footnotetext{
${ }^{14} \mathrm{http} / / /$ ja.broadcom.com/support/ethernet_nic/netxtreme_desktop.php

${ }^{15} \mathrm{http}: / /$ www.ietf.org/rfc/rfc1323.txt

${ }^{16} \mathrm{http}: / /$ ja.wikipedia.org/wiki/Microsoft_Windows_Vista
} 


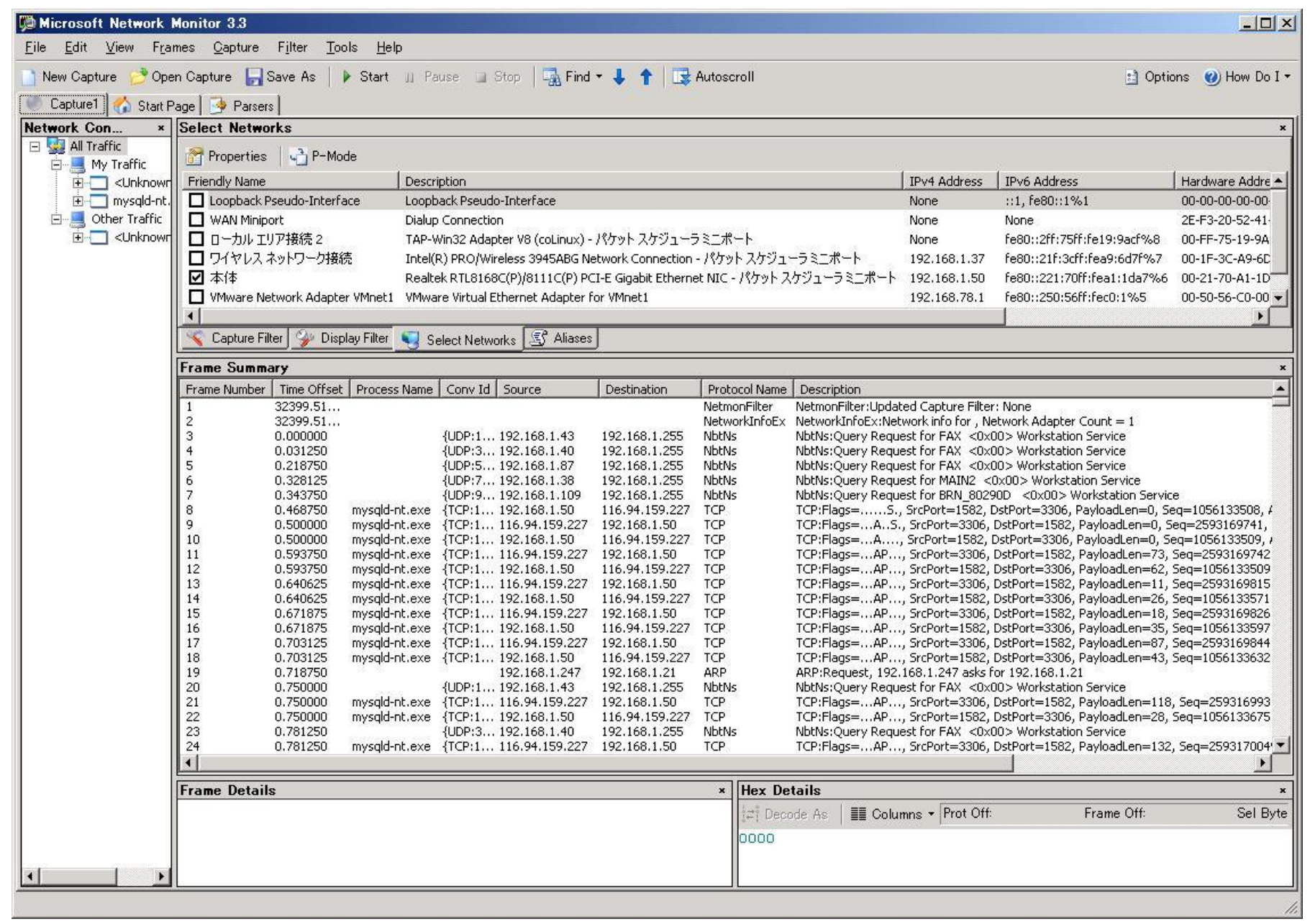

Figure 3. Screen shot image of Microsoft Network Monitor 3.3

The proposed software accelerator takes into account data compression. Namely, data packets with TCP are converted to that with User Datagram Protocol: UPD protocol ${ }^{17}$ and then transmit to the sender. Data compression can be applied to the data packets converted to UDP protocol so that the effective data rate can be improved.

\section{EXPERIMENTS}

\section{A. Experiemnt Method}

The effectiveness of the proposed software accelerator is evaluated with file transfer based on ftp protocol. Packet data flow and capacity is measured on the packet monitor with the well-known free open source software of Microsoft Network Monitor $3.3^{18}$. Figure 3 shows the screen shot image of Microsoft Network Monitor 3.3. It is confirmed that all the data packets are transmitted perfectly. By using TCP segment flag of SYN and FIN ${ }^{19}$, the start and the end time are measured. Therefore, the time required for transmission of data packets can be measured. The measured time duration is shorter than

\footnotetext{
${ }^{17}$ http://ja.wikipedia.org/wiki/User_Datagram_Protocol

$18 \mathrm{http}: / /$ www.microsoft.com/download/en/details.aspx $? \mathrm{id}=4865$

${ }^{19}$ http://en.wikipedia.org/wiki/Transmission_Control_Protocol
}

that is measured on DOS window ${ }^{20}$. Therefore, it is taken into account such duration time differences when the effective data rate is evaluated.

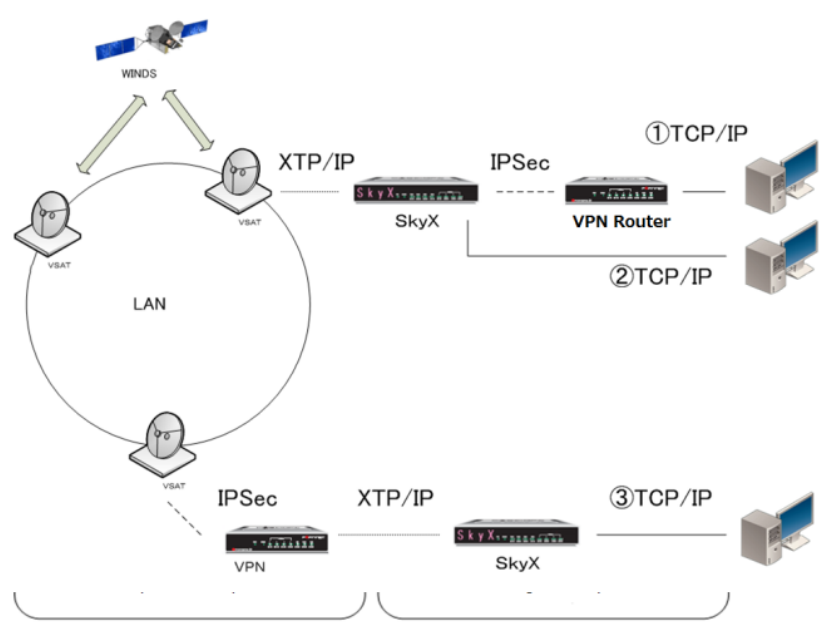

Figure 4. Network configuration

\footnotetext{
${ }^{20}$ http://www.ne.jp/asahi/hishidama/home/tech/windows/command.html
} 
Network configuration is shown in Figure 4 while experimental system configuration is shown in Figure 5, respectively. Two major terminals, Saga University and Kyushu University are connected through KIZUNA satellite. Internet connection is also confirmed as shown in Figure 6. In Figure 4, IPsec denotes Security Architecture for Internet Protocol $^{21}$, VPN denotes Virtual Private Network ${ }^{22}$

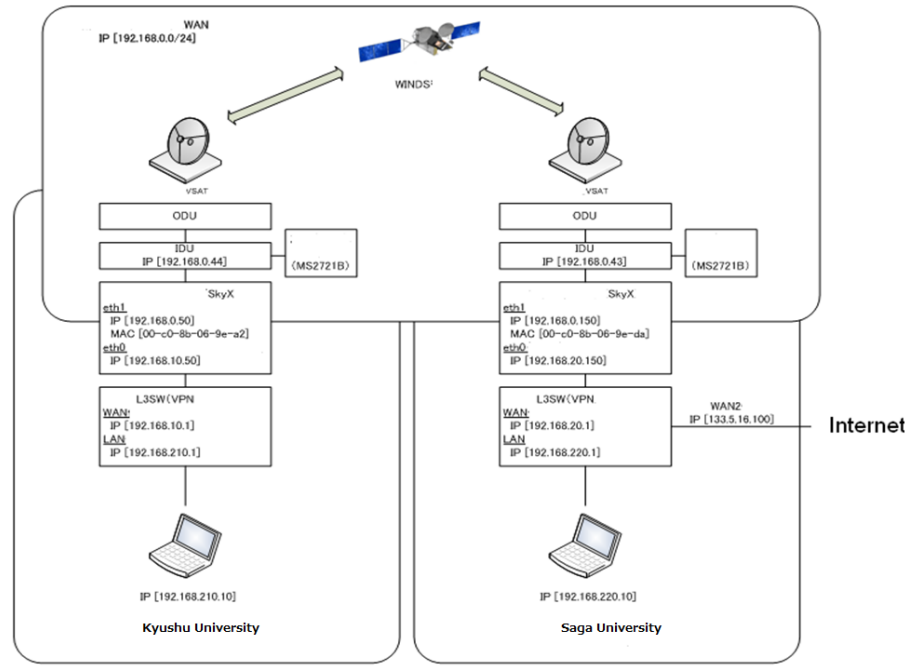

Figure 5. Experimental system configurations

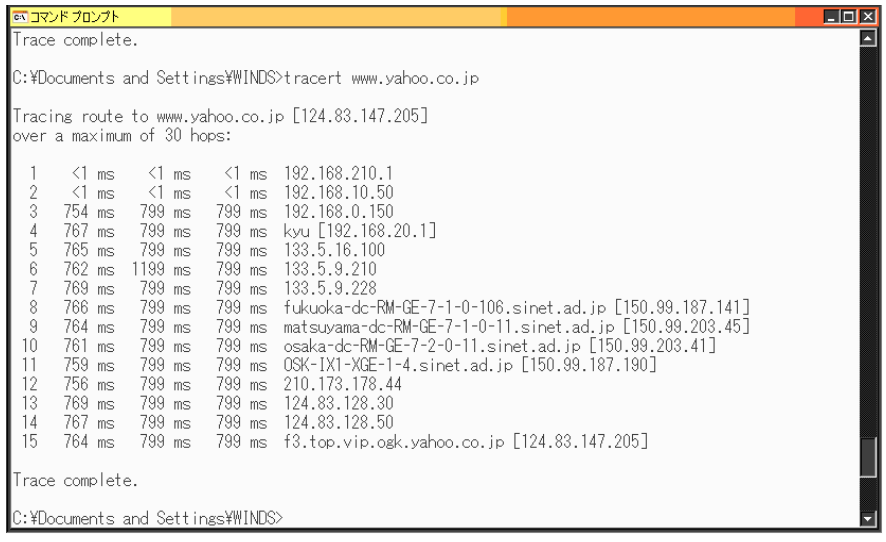

Figure 6. Internet connection confirmations

The experiments are conducted on 27 August 2008. Designated up-link data rate is $51 \mathrm{Mbps}$ between both universities. There are two datasets for the experiments, small size of (1079064Byte) and large size of (310677846Byte). These datasets are disaster relief satellite imagery data derived from Moderate resolution Imaging Spectrometer: MODIS ${ }^{23}$ and Advanced Spaceborne Thermal Emission and Reflection radiometer: $\mathrm{ASTER}^{24}$ (both mission instruments are onboard Terra satellite) as shown in Figure 7. ASTER and MODIS imagery data of Ureshino city ${ }^{25}$, Saga, Japan $\left(33^{\circ} 8^{\prime} \mathrm{N} 130^{\circ} 4^{\prime} \mathrm{E}\right)$ is acquired on February 192009.

\footnotetext{
${ }^{21}$ http://ja.wikipedia.org/wiki/IPsec

22 http://ja.wikipedia.org/wiki/Virtual_Private_Network

$23 \mathrm{http}: / /$ modis.gsfc.nasa.gov/

${ }^{24} \mathrm{http}$ ://asterweb.jpl.nasa.gov/

25 http://en.wikipedia.org/wiki/Ureshino,_Saga
}

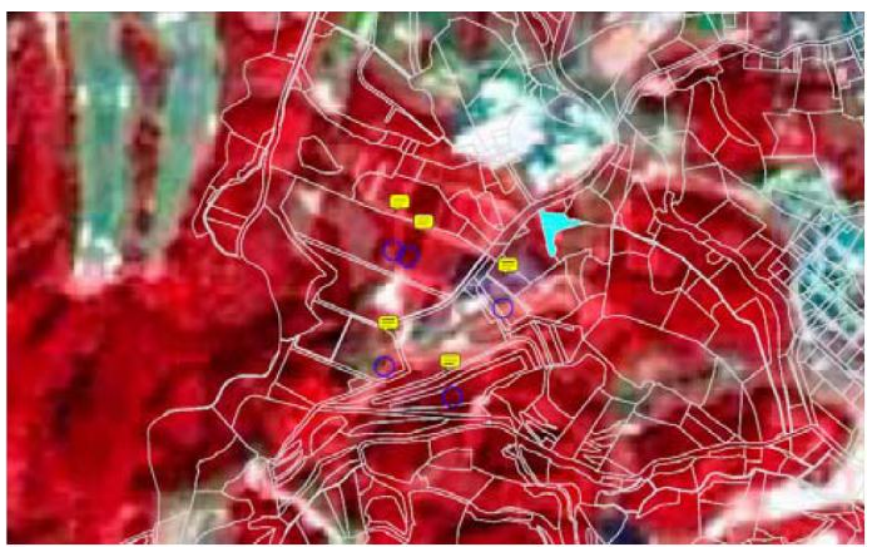

(a)ASTER imagery data of Ureshino: large size of (310677846Byte)
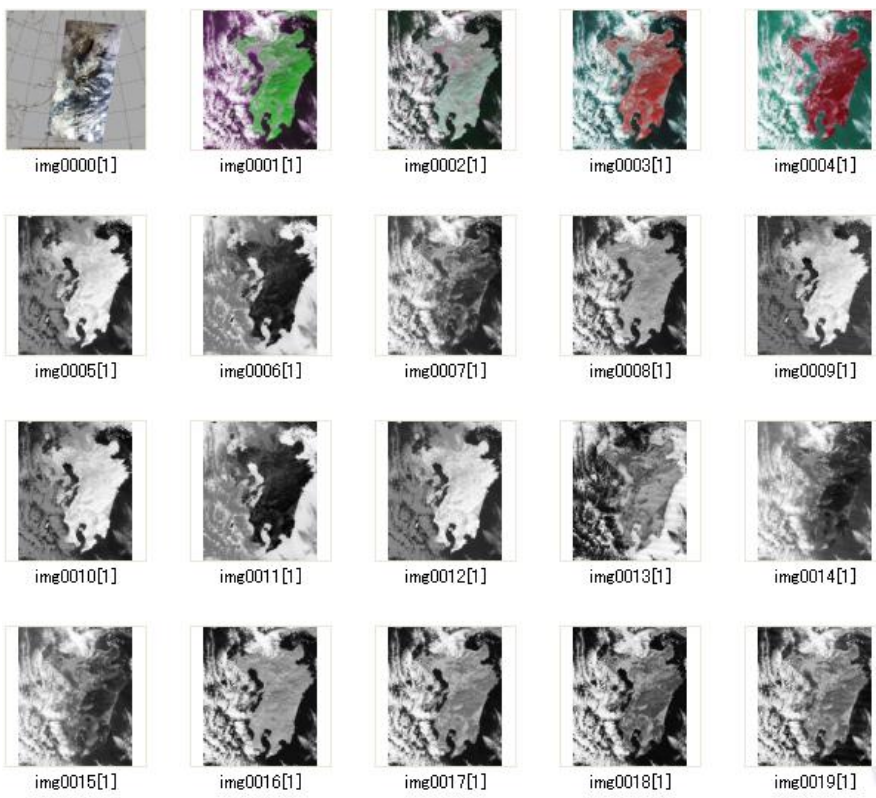

(b)MODIS imagery data of Kyushu: small size of (1079064Byte)

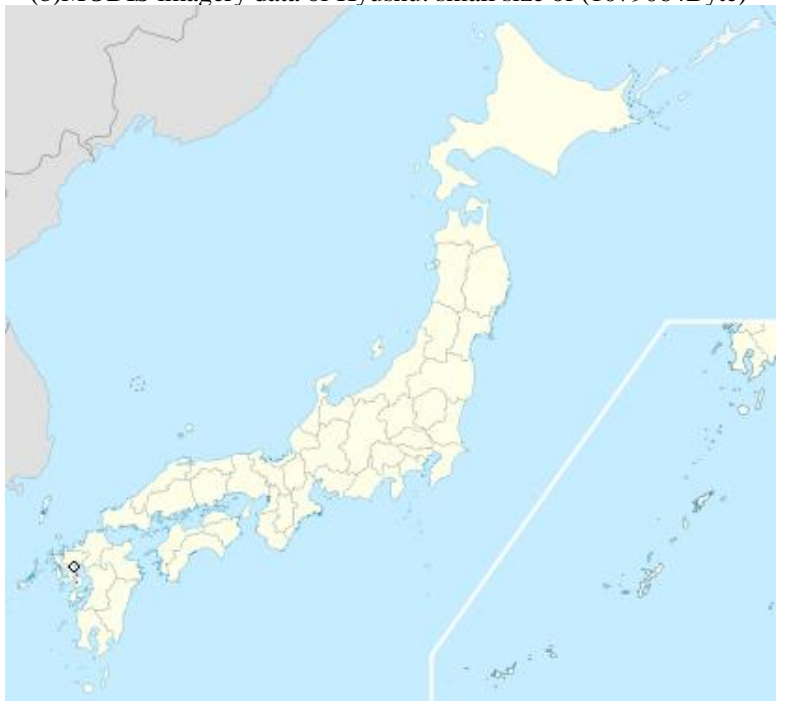

(c)Location of Ureshino and Kyushu in Japan

Figure 7. Disaster relief of ASTER and MODIS imagery data used for data transmission experiments through KIZUNA satellite 


\section{B. Experiemntal Results}

There are three major features of the proposed software accelerator, 1) acknowledge handling, 2) Maximum receiving buffer size notification, and 3) data compression. The experiments are conducted with combinations of 1), 2), and 3).

(1) Combination of 1), 2), and 3) with small dataset:

\section{$1.426757 \mathrm{sec}$}

(2) Only 1) with small dataset: $1.422575 \mathrm{sec}$

(3) Combination of 1) and 2) with large dataset: $64.688477 \mathrm{sec}$

(4) Sky-X with small dataset: $1.08 \mathrm{sec}$

(5) Sky-X with large dataset: $75.94 \mathrm{sec}$

(6) Without Sky-X (large dataset): 146.63sec, 7.36MBps

(7) Without Sky-X (small dataset): $1.33 \mathrm{sec}, 812.55 \mathrm{MBps}$

In comparison between case (1) and (2), contributions of 2) maximum receiving buffer size notification and 3) data compression are not so significant. Also it may say that 1) acknowledge handling is the most effective method for the proposed software accelerator. In comparison between case (2) and (4), the proposed software accelerator does work so as Sky-X does. Approximately, 24\% much longer time is required for the proposed software accelerator comparing to the Sky-X based hardware accelerator. It, however, depends on the data volume as well as characteristic of the dataset used for experiments. In fact, $14.8 \%$ much shorter time would be enough to send the large size of dataset for the proposed software accelerator in comparison to the Sky-X based hardware accelerator. Therefore it may concluded that the proposed software accelerator with acknowledge handling has almost same performance as Sky-X based hardware acceleration does on acceleration of data transmission even when network link includes delay of $0.8 \mathrm{sec}$.

Figure 8 shows network performance measurement data. Horizontal axis shows the different MODIS data. Figure 8 (a) shows the network performance of TCP data transmission from Saga University to Kyushu University for the case that Sky-X is off while Figure 8 (b) is that for the case of Sky-X is on. Meanwhile, Figure 8 (c) shows the network performance of UDP data transmission from Saga University to Kyushu University for the case that Sky-X is off while Figure 8 (d) is that for the case of Sky-X is on. Figure 8 (e) shows a comparison between UDP and TCP networks performances when the Sky-X is off.

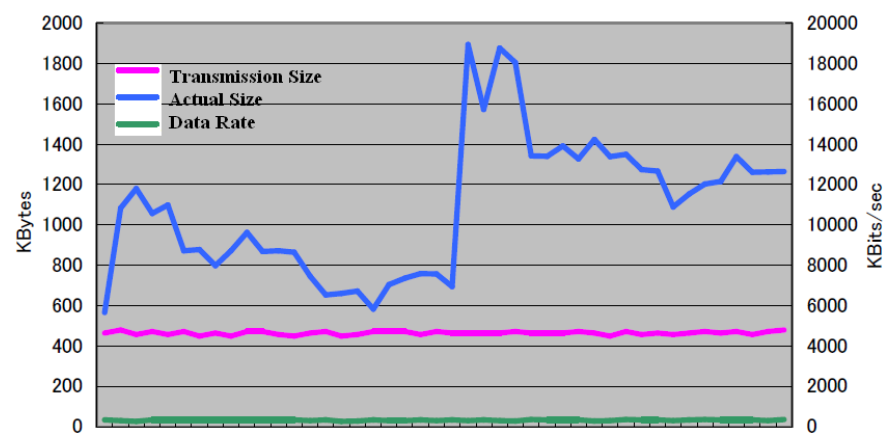

(a) TCP, Sky-X is off

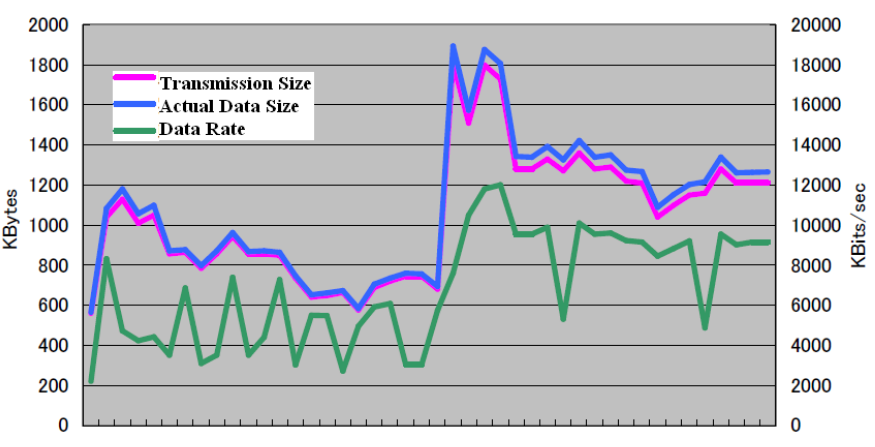

(b) TCP, Sky-X is on

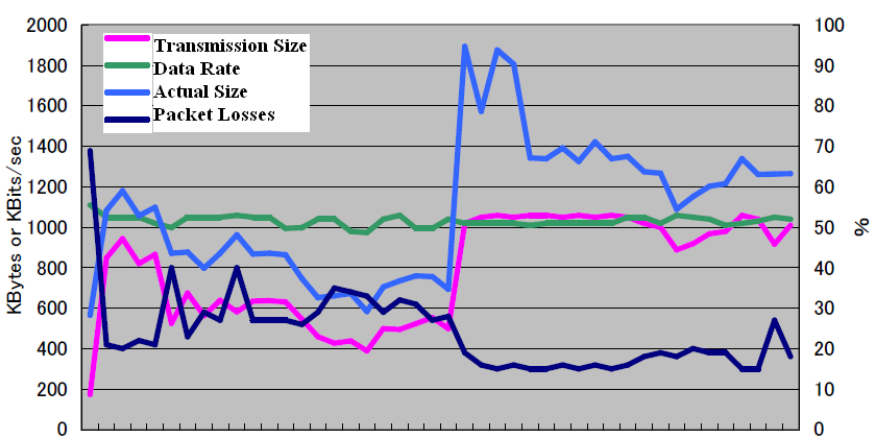

(c) UDP, Sky-X is off

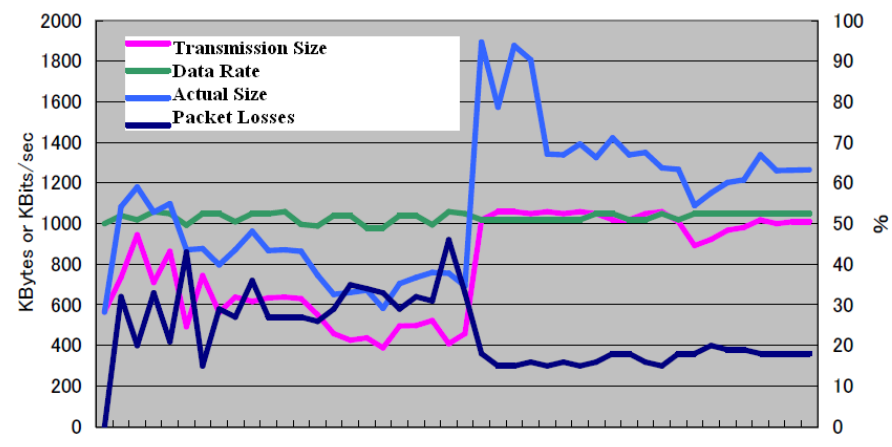

(d) UDP, Sky-X is on

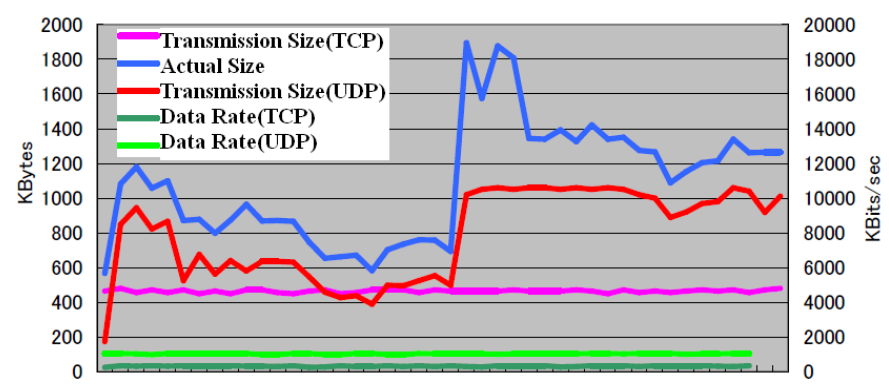

(e) Comparison between TCP and UDP when Sky-X is off

Figure 8. Network performance measured data

As a result, it is found that data rate for $S k y-X$ is on is around 100 times much larger than that for Sky-X is off.

\section{CONCLUDING REMARKS AND SOME DiscuSSIONS}

Through the experiments with University-to-University communication link through KIZUNA satellite, it is found that the proposed acknowledge handling based software accelerator is effective and is almost same as that of the widely used 
hardware accelerator. The effectiveness is much better than that of maximum receiving buffer size notification as well as data compression (conversion from TCP to UDP and application of data compression method).

The proposed acknowledge handling does not ensure TCP based data transmission so that the data transmission has to be done with the other protocols. Actually, Sky-X accelerates data transmissions based on the conversion from TCP to XTP.

Maximum receiving buffer size notification does not work in the experiments. Furthermore, RFC1323 of window size adjustment does also not work so well. Turns out, automatic window size tuning based on Windows Vista does work because it adjusts not only receiving buffer size but also sending buffer size is adjusted dynamically. The effectiveness of the automatic tuning is less than that of the proposed acknowledge handling.

Data compression does not work so well because the packet size of ftp transmission is not so large (around 1500 byte). Although data compression rate depends on the data compression method used, it cannot be compressed in some cases with a consideration of header information treatments.

Also the time required for data conversion from TCP to UDP would be a bottle neck. This method might be useful for a narrow band networks.

The delay time induced problem is affecting to not only TCP based data transmission but also the other ground based surface network links with some delay and even for mobile phone communication links. Demands on the proposed software network accelerator are getting increased in the near future.

\section{ACKNOWLEDGMENT}

The author would like to thank to Dr. Kiyotaka Fujisaki of Kyushu University, Mr. Terumasa Miyahara and Mr. Miyauchi of ELM Co. Ltd. for their great effort to conduct of the experiments as well as their valuable discussions we have had, as well as comments and suggestions.

\section{REFERENCES}

[1] Kohei Arai, Kiyotaka Fujisaki, Hiroaki Ikemi, Masato Masuya, Terumasa Miyahara, Backup communication routing through Internet Satellite, WINDS, for transmission of disaster relief data, Proceedings of the International Symposium on WINDS Application Experiments, 2010.

[2] Kohei Arai, Back-up communication link with WINDS satellite in disaster and its application ot disaster mitigation information network system, Proceedings of the SCOPE Research Results Workshop, 2010.

[3] Kohei Arai, Research and developments of a back-up communication link with WINDS satellite in disaster and its application ot disaster mitigation information network system, Proceedings of the SCOPE Research Results Workshop in 2010, 2010.

[4] Kohei Arai, Kiyotaka Fujisaki, Hiroaki Ikemi, Masato Masuya, Terumasa Miyahara, Backup communication network through WINDS satellite for disaster mitigation, Journal of the Science and Engineering Faculty, Saga University, 38, 2, 7-12, 2009.

[5] Kohei Arai, Backup communication routing through Internet satellite WINDS for transmission of disaster relief data, Proceedings of the International Symposium on WINDS, 2010

[6] Kohei Arai and Kiyotaka Fujisaki, Countermeasure for round trip delay which occurs in between satellite and ground with network accelerator, Proceedings of the Expert Meeting and the 5th Plenary Workshop of the Association for Application Experiments of WINDS, 2010.

[7] Kohei Arai, Kiyotaka Fujisaki, Hiroaki Ikemi, Ibrahim Djawaluddin, Masato Ikemi, Terumasa Miyahara, Software accelerator for high speed TCP data transmission and GIS client-server model through WINDS satellite, Journal of the Science and Engineering Faculty, Saga University, 38, 2, 13-18, 2009.

[8] Kohei Arai, Back-up communication routing through Internet satellite WINDS for transmitting of disaster relief data, International Journal of Advanced Computer Science and Applications, 2, 9, 21-26, 2011.

\section{AUTHORS PROFILE}

Kohei Arai, He received BS, MS and PhD degrees in 1972, 1974 and 1982, respectively. He was with The Institute for Industrial Science and Technology of the University of Tokyo from April 1974 to December 1978 also was with National Space Development Agency of Japan from January, 1979 to March, 1990. During from 1985 to 1987, he was with Canada Centre for Remote Sensing as a Post-Doctoral Fellow of National Science and Engineering Research Council of Canada. He moved to Saga University as a Professor in Department of Information Science on April 1990. He was a counselor for the Aeronautics and Space related to the Technology Committee of the Ministry of Science and Technology during from 1998 to 2000. He was a councilor of Saga University for 2002 and 2003. He also was an executive councilor for the Remote Sensing Society of Japan for 2003 to 2005. He is an Adjunct Professor of University of Arizona, USA since 1998. He also is Vice Chairman of the Commission "A" of ICSU/COSPAR since 2008. He wrote 30 books and published 322 journal papers. 